\title{
Constellation Design for Transmission over Nonlinear Satellite Channels
}

\author{
Farbod Kayhan \\ Politecnico di Torino \\ Dipartimento di Elettronica e Telecomunicazioni \\ 10129, Torino, Italy \\ Email:farbod.kayhan@polito.it
}

\author{
Guido Montorsi \\ Politecnico di Torino \\ Dipartimento di Elettronica e Telecomunicazioni \\ 10129, Torino, Italy \\ Email: guido.montorsi@polito.it
}

\begin{abstract}
In this paper we use a variation of simulated annealing algorithm for optimizing two-dimensional constellations with 32 signals. The main objective is to maximize the symmetric pragmatic capacity under the peak-power constraint. The method allows the joint optimization of constellation and binary labeling. We also investigate the performance of the optimized constellation over nonlinear satellite channel under additive white Gaussian noise. We consider the performance over systems with and without pre-distorters. In both cases the optimized constellations perform considerably better than the conventional Amplitude Phase Shift Keying (APSK) modulations, used in the current digital video broadcasting standard (DVB-S2) on satellite channels. Based on our optimized constellations, we also propose a new labeling for the 4+12+16-APSK constellation of the DVB-S2 standard which is Gray over all rings.
\end{abstract}

\section{INTRODUCTION}

This paper investigates the constellation design for transmission over satellite channels. Our main goal is to show that significant improvement with respect to the Amplitude Phase Shift Keying (APSK) modulations, which are currently used as the digital video broadcast (DVB-S2) standard, can be obtained by optimizing the constellation set.

In this paper we focus only on the peak power (PP) constraint as it becomes more relevant in applications with saturating high power amplifiers as a part of the communication system.

The APSK modulation has received a considerable attention for transmission over satellite channels. In particular, the radii and phases of each concentric circle of APSK constellations have been optimized in [1] as a function of signal to noise ratio (SNR) by maximizing the capacity. These optimized APSK constellations are accepted as DVB-S2 standard. One of the main reasons to focus on APSK structures is that under the PP constraint the capacity achieving distribution in the large limit of signals is proved to be discrete in amplitude (with finite number of mass points) and has a uniformly distributed phase in $[0,2 \pi)$ [2]. Even though for a finite constellation the optimal distribution is not found in general case, some previous studies indicate that APSK modulations perform very close to the symmetric capacity [3], [4].

However, many practical applications are based on the so called pragmatic approach: at the transmitter the encoder is separated from the modulator using a bit interleaver and, at the receiver, no iteration between the binary decoder and the detector is allowed. This approach implies a loss in the capacity that can be very small if a proper labeling of bits to constellation points is chosen. The relevant objective function in this case becomes the symmetric pragmatic capacity which depends on both the constellation set and the labeling [5], [6], [7], [8]. At high enough SNR, the binary reflected Gray coding has been proved to minimize the bit error probability for several modulation schemes [9], [10].

Recently a joint signal-labeling optimization scheme has been proposed by the authors [3] for designing constellations which maximize the symmetric pragmatic capacity under PP constraint as a function of signal to noise ratio (SNR) using a simulated annealing (SA) algorithm. However the simulated annealing algorithm becomes very slow for constellations with 32 signals and is practically infeasible for higher order constellations. This is mainly due to two facts. First of all, the complexity of SA algorithm grows quadratic with respect to the number of constellations points. Secondly, one usually needs to use a slower cooling scheduling in order to reach a good local maximum by increasing the constellation's cardinality. In order to speed up the SA algorithm, we introduce a symmetry condition over the constellation points.

We compare the performance of our optimized constellations with 32-APSK modulation used in DVB-S2 standard. We consider both static and dynamic pre-distortion techniques in order to reduce the warping effects and/or inter-symbol interference (ISI) of the non-linear channel. Our optimized constellation outperforms the DVB-S2 current standard up to $0.5 \mathrm{~dB}$. Inspired by our optimized constellations, we also propose a new labeling for the 4+12+16-APSK constellation which is Gray over all rings. This new mapping results in a gain of approximately $0.15 \mathrm{~dB}$ with respect to the current DVB-S2 labeling for all system scenarios.

The rest of this paper is organized as follows. Section [II describe the considered realistic satellite non linear system. In section [III we describe the main notations and formulate the optimization problem by introducing some simplifications. A short description of the optimization algorithm and its improved version with the symmetry condition is provided in section IV The optimization results are presented in section $\mathrm{V}$ We also provide a new Gray labeling for $4+12+16-\mathrm{APSK}$. 
Finally, in section VI] we compare the performance of our optimized constellation with 32-APSK constellation on the realistic scenario described in Section П

\section{SySTEM Model}

In Figure 1 we show the system model considered in this paper. At the transmitter side, the information bits are passed to the channel encoder. The codewords then are interleaved and mapped into the modulation signals. After passing through a squared root raised cosine (SRRC) filter, the signal is sent to the satellite transponder. In this model we ignore the uplink noise. The transponder model is composed of an input demultiplexer (IMUX) filter, a travelling wave tube amplifier (TWTA) and an output multiplexer (OMUX) filter [11]. The downlink noise is modelled as the complex AWGN (CAWGN). At the receiver, the signal is first passed through the SRRC filter. Note that due to the non-linear characteristics of the channel, this filter is no longer matched, and therefore ISI is introduced. The decoder receives the soft estimated bits from demapper/deinterleaver and calculates the a posteriori probabilities without any further interaction with demodulator and demapper.

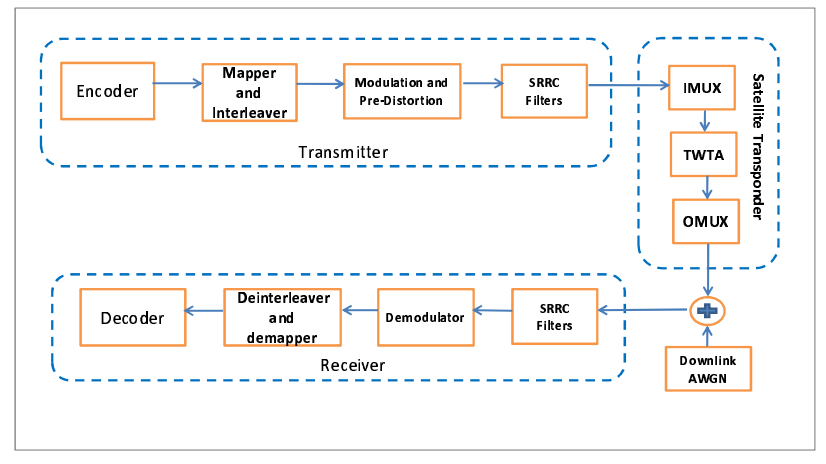

Fig. 1. Satellite communication system model.

\section{A. High Power Amplifiers and Pre-Distortion Techniques}

Nonlinear characteristics of the satellite channel largely depend on the HPA used at the satellite transponder and operating close to the saturation point. The HPA nonlinearity changes both the amplitude and relative positions of the constellation points. These changes are described with AM/AM and $\mathrm{AM} / \mathrm{PM}$ curves depicted in figure 2.

In some systems, the nonlinearity distortions are reduced by using a pre distorter at the transmitter [12], [1] and [13]. We distinguish three main categories of pre distortion techniques as follows:

Static symbol level predistortion: This technique modifies the location of transmitted constellation points on the complex plane with respect to their nominal positions in order to compensate the effects of the nonlinearity. The static symbol level pre distorter does not modify the spectrum of transmitted signal.

Dynamic symbol level predistortion: Static symbol level predistortion does not consider the impact of memory introduced

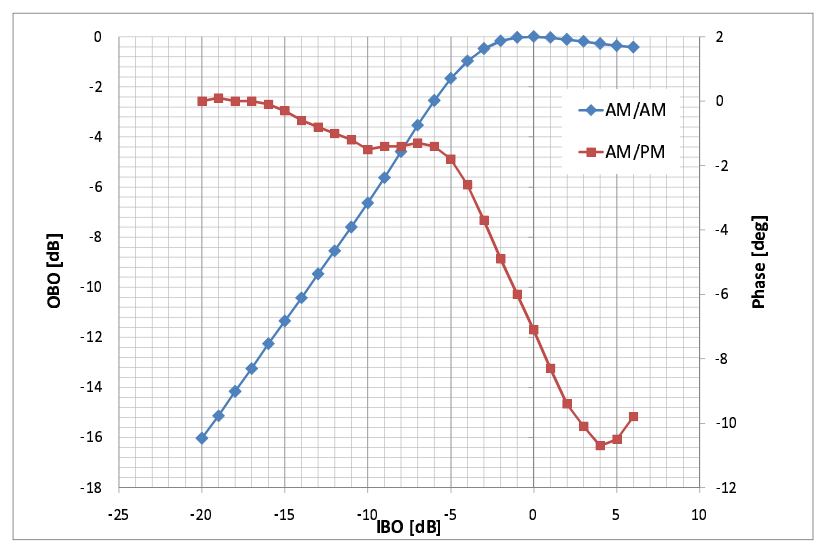

Fig. 2. AM/AM and AM/PM characteristics of DVB-S2 non linear TWTA.

by the filters in the system and correspondingly neglects the ISI introduced by neighboring symbols. In order to combat the ISI, one may takes into account also the effect of some previous and future symbols. The dynamic pre-distorter algorithms consider also the memory of the channel in order to compensate for the clustering phenomenon. Several dynamic pre-distorters have been proposed in the literature [1] and [14]. Static sample level predistortion: This technique pre distorts the signal after the transmitter shaping filter, working at several samples per symbol. This technique is essentially equivalent to an HPA linearizer and can be applied only if the predistorter acts immediately before the HPA. In the following we refer to the Soft Limiter or Ideal amplifier model, which corresponds to the use of an ideal sample level pre-distorter. In this case, the AM/AM curve is linear up to the saturating point and remains constant afterwards.

APSK constellations are more robust to the distortions caused by nonlinear amplifier with respect to Quadrature Amplitude Modulation (QAM). In general the power efficiency of APSK modulation schemes can be improved by applying pre-distortion on the transmitted signals. Despite the hardware complexity impact, pre-distortion techniques have been already adopted in systems implementing standards including APSK constellation such as the DVB-S2 [12].

\section{STATEMENT OF THE OPTIMIZATION PROBLEM}

We consider a complex constellation $\chi$ with $M=2^{m}$ elements. The elements of $\chi$ are referred as constellation points or simply signals. The Euclidean distance between two points in the complex plane is denoted by $d(.,$.$) , and d_{H}(.,$. is used to denote the Hamming distance between two binary sequences.

The signals are associated to the bits at the input of the modulator through the one-to-one labeling $\mu: \chi \rightarrow\{0,1\}^{m}$. In particular, for any given signal $x, \mu^{i}(x)$ is the value of the $i^{\text {th }}$ bit of the label associated to it. A labeling for $\chi$ is called a Gray mapping if for any two signal $x_{i}, x_{j} \in \chi$ we have $d_{H}\left(\mu\left(x_{i}\right), \mu\left(x_{j}\right)\right)=1$ if $d\left(x_{i}, x_{j}\right) \leq d\left(x_{i}, x_{k}\right)$, for all $x_{k} \in \chi$. 
Even though the separation of detection and decoding described in section $\Pi$ is in general suboptimal, it is widespread in communications applications due to the complexity reduction at the receiver [6], [7], [8]. The loss in terms of channel capacity compared to optimal joint detection and decoding, not only depends on the constellation, but also on the labeling. In general, non-Gray mappings induce a higher loss of capacity at high SNR's. For a given constellation $\chi$ and labeling $\mu$, the symmetric pragmatic capacity of the channel is defined as

$$
\mathcal{C}(\chi, \mu)=\sum_{i=1}^{m} I\left(\mu^{i}(X) ; Y\right),
$$

where $\mu^{i}(X)$ is the random variable indicating the $i^{t h}$ bit associated to the transmitted signal $X, Y$ is the received symbol, and $I(. ;$.$) denotes the mutual information function.$ Notice that symmetric pragmatic capacity assumes uniform probability of the input symbols so that it can be equivalently named symmetric pragmatic mutual information.

\section{A. Assumptions for the Optimization Problem}

In order to simplify the optimization problem we make the following assumptions to model the system described in Section [II First of all we ignore the effect of filtering. The channel is modelled as $y_{k}=f\left(x_{k}\right)+n_{k}$ where $x_{k}$ is the transmitted constellation point, $n_{k}$ is the additive Gaussian noise and $f$ represents the memoryless effect of the non linearity (AM/AM and AM/PM curves). Moreover, we assume that the non-linearity is the Soft Limiter, corresponding to the assumption of using an ideal sample level predistorter:

$$
|f(x)|=\min \left(|x|, P_{\text {sat }}=1\right), \quad \arg (f(x))=\arg (x),
$$

where $P_{\text {sat }}$ is the saturated power of the amplifier. With this assumptions the peak-power limit imposed over the constellation becomes equivalent to the constraint on $P_{\text {sat }}$. In sectionVI we will validate the above assumptions by testing the resulting optimized constellations in realistic scenarios.

\section{Simulated Annealing Algorithm for Joint SigNAL/LABELING OPTIMIZATION}

In [3] a simulated annealing (SA) algorithm has been used for maximizing $\mathcal{C}(\chi, \mu)$. SA, under some conditions on the cooling schedule, guarantees convergence to the global optimum even in non convex problems and thus it is preferable to other local optimization techniques like the gradient algorithm. Here we briefly review the SA algorithm presented in [3]. For some other applications of SA algorithm in coding theory we refer the readers to [15] and the references therein.

At any time step $t$, we randomly choose a constellation point and move it into a random new position inside the unitary circle. If the capacity increases by this change, we accept the new constellation. On the other hand, if the capacity decreases, the new constellation is accepted only with probability $e^{-\left|\Delta C_{t}\right| / T_{t}}$, where $\Delta C_{t}$ is the difference between the capacity of constellations at times $t$ and $t-1$, and $T_{t}$ is the "temperature" at time $t$, a parameter of the SA algorithm. The initial and final temperatures are fixed at the beginning and the cooling parameter, $0<\alpha<1$, indicates the cooling scheduling, i.e., $T_{t+1}=\alpha T_{t}$.

The main adaptation needed to speed up the SA algorithm for maximizing the capacity is to define a maximum displacement length as a function of time. For details on such adaptation we refer the readers to [3] and the references within. Note that the initialization step distributes uniformly the $M$ signals in the unitary circle. When the cost function is the symmetric pragmatic capacity, at the beginning of the algorithm an arbitrary labeling is also assigned to each point and never changed afterwards, so that constellation points "move" together with their labels during the optimization.

The output of this algorithm -with the chosen parametersis independent of the initial conditions for constellations with up to 16 signals, suggesting that the algorithm is optimal for such small constellations. However for larger constellations the SA algorithm becomes very slow, and practically not applicable. Furthermore its output starts depending on the initial conditions.

\section{A. A Simplification of Simulated Annealing with Symmetry Condition}

In order to reduce the complexity of the SA algorithm, we introduce a symmetry condition over the constellation points. In particular we suppose that the constellation is symmetric with respect to reflections on both horizontal and vertical axis. To adapt the algorithm with this symmetry condition we confine the optimization to the first quadrant of the unitary circle, i.e., we suppose that exactly one forth of points have positive coordinates and we assume that the remaining points are obtained by reflection with respect to the two axis. We start the algorithm by random positioning of $M / 4$ signals in the first quadrant. When the objective function is the symmetric pragmatic capacity we also assign a random labeling of length $m-2$. The labeling associated to reflected points is the same as the corresponding signal in first quadrant, with two additional bits selecting the quadrant. Note that if two points have Hamming distance $d$ in the first quadrant, the corresponding reflected points also have the Hamming distance $d$ in all other three quadrants.

The optimization technique is similar to the case without symmetry condition. At each step of the SA we choose randomly a point from the first quadrant and move it into a new random position inside this quadrant. Note that this change cause the simultaneous change of the three points in the other quadrants due to the symmetry condition. In other words, at each step of SA, four points are simultaneously moved inside the unitary circle.

With the addition of the symmetry condition the time needed to converge to a good local maximum reduces from several days to only several minutes. Constellations obtained with this additional constraint have the same capacity as those obtained without it and perform exactly the same over the nonlinear satellite channel. Furthermore, the algorithm becomes independent of the initial conditions (over ten runs). This 
strongly suggests that the obtained constellations are optimal under the given conditions.

\section{Optimization Results}

In this section we present the constellations optimized by the SA algorithm with symmetry condition. As we have mentioned, we are interested in optimizing the constellations under the peak-power constraint and therefore in what follows we always fix the maximum power to one $\left(|x|^{2} \leq 1\right)$. More over, instead of the signal to noise ratio, we consider the peak power to noise ratio (PSNR) as a measure for comparing our constellations. Note that PSNR is the ratio between the peak power of the constellation and the noise power spectral density

$$
\mathrm{PSNR} \triangleq \frac{1}{N_{0}} \geq \mathrm{SNR} \triangleq \frac{1}{M} \sum_{x \in \chi} \frac{|x|^{2}}{N_{0}}
$$

Under the assumptions of Section $\amalg$ II-A the PSNR coincides with the ratio $P_{s a t} / N_{0}$ so that

$$
\mathrm{SNR}=\frac{E_{s}}{N_{0}}=\frac{P_{\mathrm{sat}}}{N_{0}} \cdot \frac{E_{s}}{P_{\mathrm{sat}}}=\mathrm{PSNR}-O B O[\mathrm{~dB}] .
$$

Here we present two constellations optimized for two different values of PSNR. Figure 3 shows the constellation obtained for PSNR $=15$. The mapping is presented only in the first quadrant by numbers form zero to seven. The actual mapping of a given signal in this quadrant is a sequence of 5 bits, of which the first two bits are zero and the last three bits correspond to the integer associated to it. For example the point with label "7" has the mapping "00111". The mapping for signals in other quadrants are obtained by symmetry and change of the first two bits selecting the quadrant. Notice that the constellation in 3 is by no means optimal in the classical "minimum distance" sense. At moderate and low SNR indeed the minimum distance is not dominant in the expression of the mutual information.

In Figure 4 we show the constellation obtained for PSNR = 18 This constellation is optimized for a wide range of high PSNR values and will be used in the simulations reported in Section VI

In Figure 5 we plot the capacity curves of the two optimized constellations as a function of PSNR and compare them with the 4+12+16-APSK modulation used currently as the DVBS2 standard. As it can be seen, a gain of approximately 0.5 $\mathrm{dB}$ is obtained by our optimized constellations. In Section VI we will investigate whether this gain is preserved also in more realistic scenarios including the presence of the HPA amplifier.

The main question which arises by adding the symmetry condition over constellation space is its impact on the optimality of obtained constellations. We conjecture that the optimal constellations may not satisfy the symmetry condition. The reason is mainly because the symmetry condition implies that the number of signals on the outer ring be divisible by four. This condition may not need to be true in general case for an optimized constellation (see also [3]).

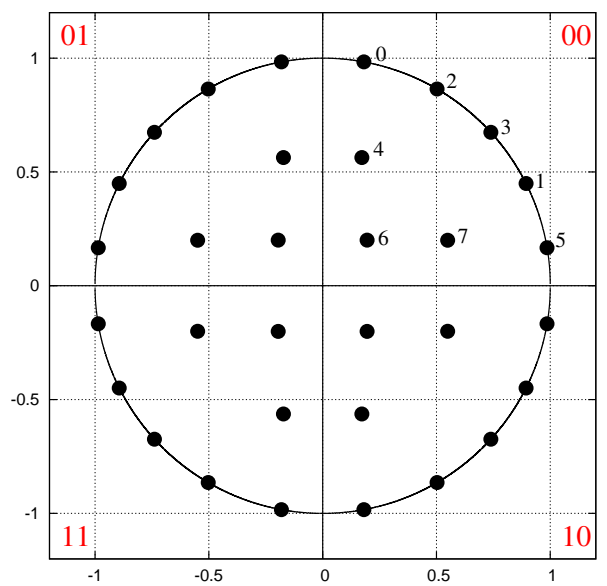

Fig. 3. Optimized constellation at PSNR $=15$ with the symmetry condition.

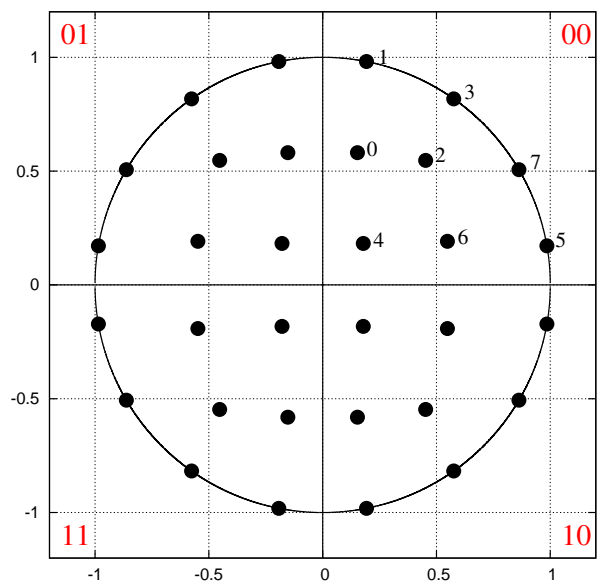

Fig. 4. Optimized constellation at PSNR $=18$ with the symmetry condition.

Note that one may change the ordering of the bits of a given mapping without effecting the symmetric pragmatic capacity in equation 1 When the interleaver and coding is random the ordering of the bits of a given mapping is not an issue. However, in DVB-S2 standard a structured row-column interleaver followed by a particular LDPC code is used and therefore it is important to optimize also the placement of the bits [11]. This optimization can be done separately after the joint signal/labeling optimization.

\section{A. Gray Mapping for 4+12+16-APSK}

Even though the constellation in Figure 4 has not APSK structure, its mapping can be adopted by $4+12+16-$ APSK constellation. Unlike the current mapping of the DVB-S2 standard, this mapping is Gray over all rings. This new mapping for 32-APSK constellation is shown in figure 6 Note that we report the results obtained for this new mapping using the 4+12+16-APSK constellations with optimized radii and phases in [11] and therefore only the mapping is different from the standard.

In Figure 5 we also plot the symmetric pragmatic capacity curve for the $4+12+16$-APSK constellation with the Gray 


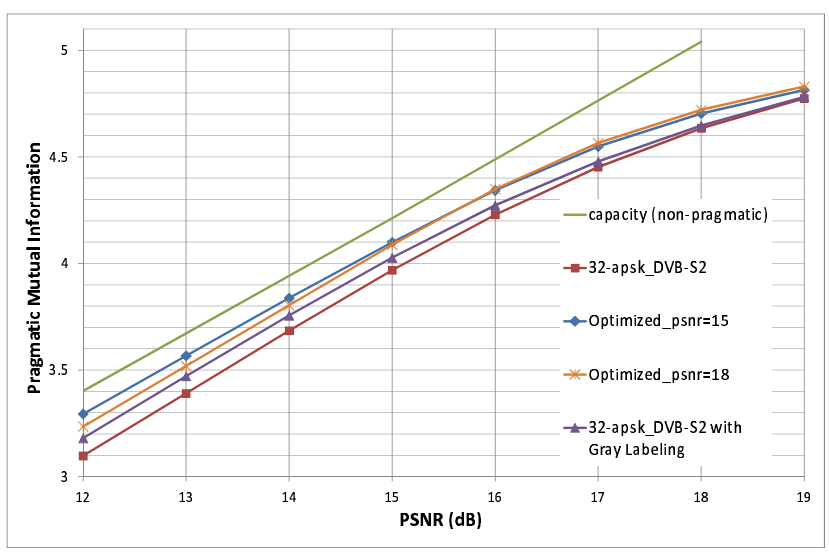

Fig. 5. Symmetric pragmatic mutual information for the optimized constellations as a function of PSNR. A gain of approximately $0.5 \mathrm{~dB}$ is obtained with respect to the 32-APSK modulation scheme used currently in DVB-S2 standard.

labeling of figure 6 . This constellation has a larger pragmatic capacity than the DVB-S2 standard constellation as a function of both SNR and PSNR. Therefore, we expect that this constellation performs better than the current standard in all cases. This is confirmed by our simulations over both linear and non-linear channels showing a gain of approximately 0.15 $\mathrm{dB}$ in all system scenarios. As we will see in the next section, the optimized constellation performs even better than the 32APSK with Gray labeling, suggesting that the gain obtained by the optimization is not only due to a better mapping but also the shape of the constellation.

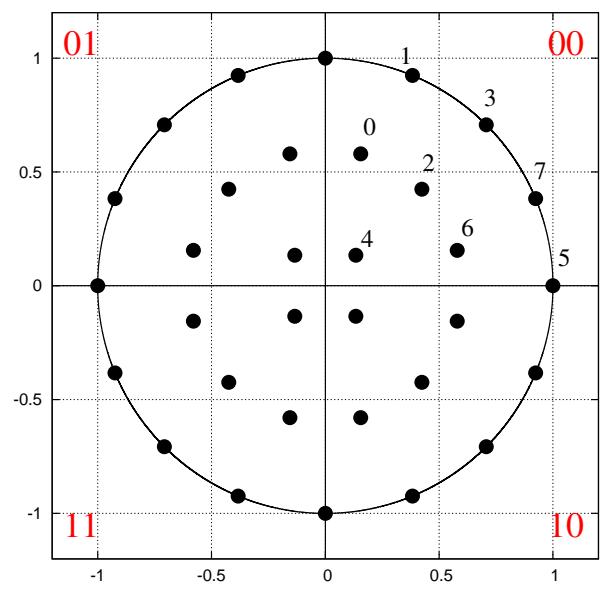

Fig. 6. New labeling for $4+12+16-$ APSK constellation, inspired by the mapping of the optimized constellation in figure 4 Note that this mapping is Gray over all rings.

\section{Simulation Results}

In this section we investigate the performance of our optimized constellations over a realistic system model. Systems with and without pre-distortion techniques have been considered. We compare our results with the DVB-S2 standard 32-APSK constellation. In all simulations we have used a LDPC code of rate $R=3 / 4$ and length $N=64800$. The roll-off factor of the SRRC filter is fixed to $\alpha=0.2$ and the normalized transponder bandwidth (ratio between symbol rate and $3 \mathrm{~dB}$ bandwidth of transponder) is $\phi=0.8$. We use the optimized constellation in figure 4 in all the simulations and refer to it as the optimized constellation. In all cases the optimal Input Back-Off (IBO) values are found preliminary and are used for simulating the bit error rates.

In our simulation results we choose the PSNR as the measure of the quality of the channel instead of conventional SNR. Indeed, the choice of SNR can be misleading when comparing performance for different IBO as it only captures the loss of performance due to the intersymbol interference (ISI) introduced by the non linearity, but it neglects the power loss at the receiver induced by the transponder Output BackOff $(\mathrm{OBO})$. If one is interested in finding the corresponding average SNR, it should subtract the value of OBO for each simulated point. Notice that since the IBO is optimized for each given PSNR the correspondence between the two quantities is not a simple offset.

\section{A. Performance without Pre-Distortion Techniques}

We first investigate the performance of our optimized constellation over a system where no pre-distortion technique is present. In figure 7 we compare the BER of the optimized constellation with 32-APSK for the two different systems:

1) A system without the satellite transponder in the communication chain, where the channel is linear with AWGN noise;

2) A system with non-ideal HPA in the communication chain but with no pre-distortion technique available at the transmitter.

As it was expected, the gain observed in figure 5 is maintained for the system with no HPA, however this gain is reduced into approximately $0.2 \mathrm{~dB}$ for the system with non-ideal HPA. As we will see in the next subsection, this gain can be increased by applying some pre-distortion techniques.

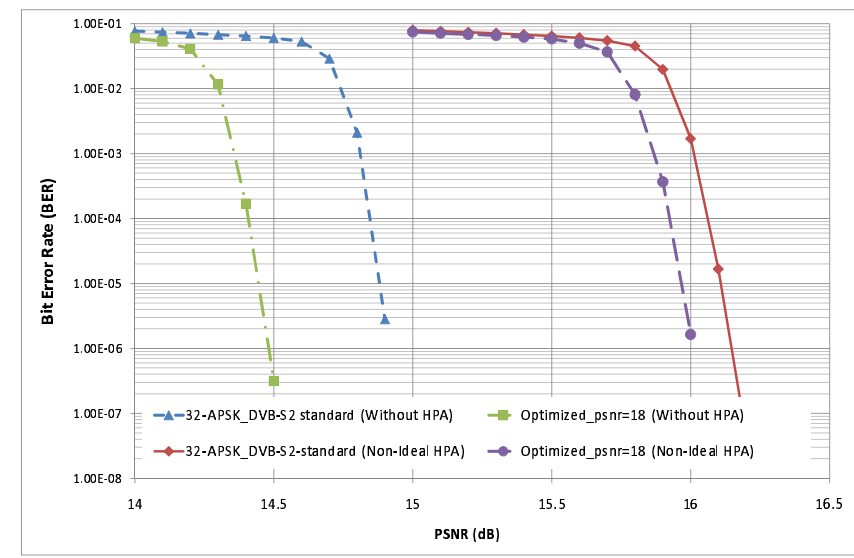

Fig. 7. Comparison between the optimized constellation and the 32-APSK modulation of DVB-S2 standard in the presence of non-ideal HPA with no predistortion. Also the simulation results for a system with no HPA is presented. 


\section{B. Performance with Pre-Distortion Techniques}

In this section we simulate the performance of our optimized constellation over systems where a pre-distortion technique is available at the transmitter. All three pre-distortion techniques discussed in subsection $\amalg-\mathrm{A}$ are considered. For details on the implementation of static and dynamic pre-distortion algorithms we refer the readers to [14]. For dynamic predistortion we have considered $L=3$ symbols simultaneously for reducing the ISI introduced by the non-linear HPA.

In figure 8 we present the BER for both the optimized constellation and the 32-APSK for all discussed pre-distortion techniques. The results show that the gain with respect to the non-ideal HPA is increased only when a dynamic predistorter is used, implying that the optimized constellations suffer more from the ISI. In this case the gain with respect to the DVB-S2 standard is slightly more than $0.3 \mathrm{~dB}$. The gain which can be obtained by using the Gray labeling for the 32APSK constellation instead of the DVB-S2 standard labeling is around $0.15-0.2 \mathrm{~dB}$ in all cases. Notice that dynamic predistortion can outperform the "ideal" HPA. This is due to the fact that dynamic predistortion also reduce ISI while the ideal HPA is a memoryless device that cannot reduce ISI.

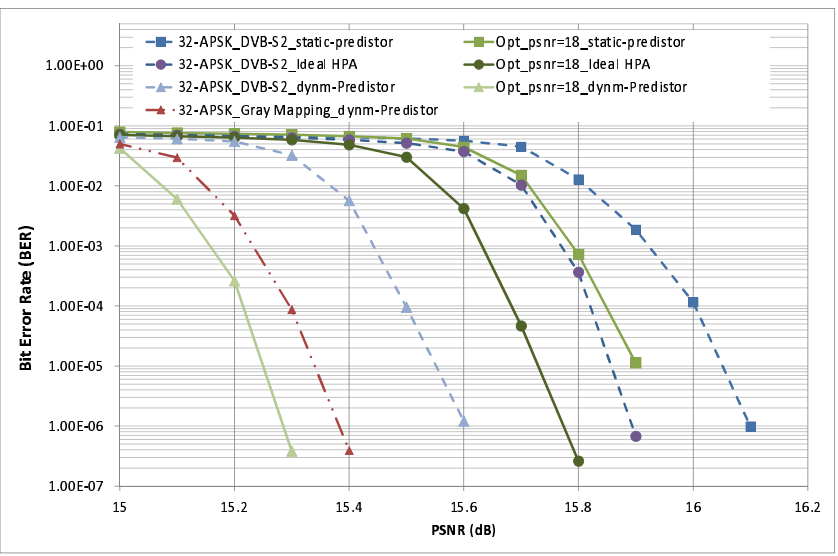

Fig. 8. Comparison between the optimized constellation and the DVB-S2 standard 32-APSK modulation scheme in a system with pre-distortion. Static, dynamic and ideal pre-distortion techniques have been considered.

\section{CONCLUSIONS}

In this paper we have proposed a simulated annealing algorithm to optimize the constellation space under the peakpower constraint for constellations with 32 signals. We have introduced a symmetry condition which speeds up simulated annealing algorithm, allowing to optimize the constellations with up to 64 signals. We have compared the performance of our optimized constellations with 32-APSK modulations used in DVB-S2 standard in a realistic scenario. Systems with both static and dynamic pre-distorters have been considered. Depending on system characteristics a gain of 0.2 to $0.5 \mathrm{~dB}$ can be obtained with respect to the current DVB-S2 standard by using the optimized constellations. We have also proposed a Gray labeling for $4+12+16$-APSK constellation. A gain of approximately $0.15 \mathrm{~dB}$ with respect to the current labeling of DVB-S2 standard can be obtained with this new mapping.

\section{APPENDIX I: GRAY MAPPINGS FOR APSK CONSTELlations}

In the large SNR region the capacity of a constellation mainly depends on the minimum distance [16]. In such scenarios, given a constellation it is desirable to find a Gray mapping for the constellation. For a given APSK constellation the problem of finding a Gray mapping is not solved in general case. However it is easy to find a mapping which is Gray over each ring of the APSK constellation if all rings contain an even number of signals. If all points are nearer to a point lying on the same ring than the points over other rings then having a Gray mapping over all rings is desirable in the limit of large SNR (PSNR).

Lemma 7.1: An APSK constellation with $2^{m}$ signals admits a labelling which is Gray over all rings if and only if each ring contains an even number of points.

Proof: It is easy to show that no Gray mapping exists for an odd number of points over a ring and therefore it is necessary to have an even number of points over each ring of a given APSK constellation. To proof the other side of the lemma, we explicitly construction a mapping which is Gray for any given integer partition of $2^{m}$. Let $2^{m}=n_{1}+n_{2}+\ldots+n_{p}$ be a given partition of $2^{m}$ where $p$ is arbitrary integer and $n_{i}$ is even. First we list in a column all the sequences with $m-1$ bits $\left(2^{(m-1)}\right.$ binary numbers) such that each sequence has Hamming distance one from its neighbours. Then partition this list into $p$ subsets each having $n_{i} / 2$ elements for $i=$ $1 \ldots p$. Duplicate the sequences of each subset by adding a bit (both zero and one) at the beginning of each existing element. Now we have $p$ subsets each having exactly $n_{i}$ elements. The sequences in each subset can be then arranged over a circle in such a way that the induced mapping is Gray.

The proof can be understood easily by looking at a simple example. Suppose that we want to find a Gray mapping a $4+6+8+14$-APSK constellation. Such a mapping is provided in table I The sequences in each section (separated by a horizontal line) can be used to have a Gray mapping for the ring with the corresponding number of signals.

In a realistic situation with medium SNR or PSNR, one may need to have also a good distance property between the rings of a given APSK constellation. In this case one may first obtain a Gray mapping over each rings as it was explained above and then calculate the capacity of all possible rotations of rings choosing the rotation with highest pragmatic capacity. However this method may not be optimal in general case and becomes computationally infeasible for large constellations.

\section{ApPEndix II: Higher ORder CONSTEllations}

As we have mentioned our optimization technique can be used to optimize higher order constellations in two or more dimensions. For example in figure 9 we show the output of the algorithm for a constellation with 64 signals optimized at PSNR $=18$. As it can be seen, the inner points have a cross 32-QAM structure while 32 remaining point lie on the outer 


\begin{tabular}{|lllll|}
\hline 0 & 0000 & & 1 & 0000 \\
0 & 0001 & & 1 & 0001 \\
\hline 0 & 0011 & & 1 & 0011 \\
0 & 0010 & & 1 & 0010 \\
0 & 0110 & & 1 & 0110 \\
\hline 0 & 0111 & & 1 & 0111 \\
0 & 0101 & & 1 & 0101 \\
0 & 0100 & & 1 & 0100 \\
0 & 1100 & & 1 & 1100 \\
\hline 0 & 1101 & & 1 & 1101 \\
0 & 1111 & & 1 & 1111 \\
0 & 1110 & & 1 & 1110 \\
0 & 1010 & & 1 & 1010 \\
0 & 1011 & & 1 & 1011 \\
0 & 1001 & & 1 & 1001 \\
0 & 1000 & & 1 & 1000 \\
\hline
\end{tabular}

TABLE I

A MAPPING WHICH IS GRAY OVER EACH RING OF A $4+6+8+14$-APSK CONSTELLATION FOLLOWING THE CONSTRUCTION IN LEMMA 7.1

ring and the associated mapping is Gray. This constellation performs slightly (approximately $0.15 \mathrm{~dB}$ ) better than the $4+12+18+30$-APSK constellation for a LDPC code with rate $R=3 / 4$ and length $N=64800$ over the non-linear channel with ideal amplifier. More research on this direction is on going.

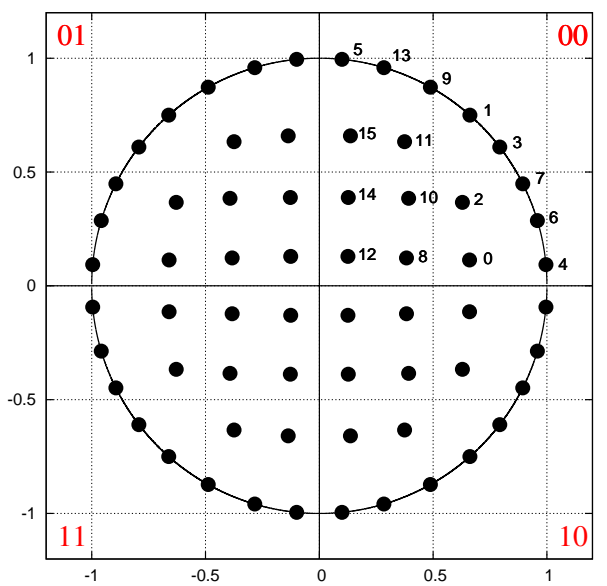

Fig. 9. Optimized constellation at PSNR $=18$ with the symmetry condition.

\section{ACKNOWLEDGMENT}

The authors wish to thank Sergio Benedetto, Nader Alagha and Riccardo De Gaudenzi for several discussions and useful suggestions. This work was performed under the ESA/ESTEC contract n. 4000102300, "Enhanced Digital Modem Techniques Development and Validation".

\section{REFERENCES}

[1] R. De Gaudenzi, A. Guillen i Fabrigas, and A. Martinez, "Performance analysis of turbo-coded apsk modulations over nonlinear satellite channels," IEEE Transactions on Wireless Communications,, vol. 5, no. 9, pp. 2396-2407, September 2006.

[2] S. Shamai and I. Bar-David, "The capacity of average and peakpower-limited quadrature gaussian channels," IEEE Transactions on Information Theory, vol. 41, no. 4, pp. 1060-1071, Jul 1995.

[3] F. Kayhan and G. Montorsi, "Joint signal-labeling optimization for pragmatic capacity under peak-power constraint," Proceedings of IEEE Global Telecommunications Conference, Dec. 2010.

[4] S. Ikeda, K. Hayashi, and T. Tanaka, "Capacity and modulations with peak power constraint," arXiv:1005.3889 [cs.IT], May 2010.

[5] G. Ungerboeck, "Channel coding with multilevel/phase signals," IEEE Transactions on Information Theory, vol. 28, no. 1, pp. 55-66, 1982.

[6] A. J. Viterbi, J. K. Wolf, E. Zehavi, and R. Padovani, "A pragmatic approach to trellis-coded modulation," IEEE Communications Magazine, vol. 27 , no. 7, pp. 11-19, July 1989 .

[7] G. Caire, G. Taricco, and E. Biglieri, "Bit-interleaved coded modulation," IEEE Transactions on Information Theory, vol. 44, pp. 927-946, May 1998.

[8] R. Muller and W. Gerstacker, "On the capacity loss due to separation of detection and decoding," IEEE Transactions on Information Theory, vol. 50, no. 8, pp. 1769-1778, Aug. 2004.

[9] E. Agrell, J. Lassing, E. G. Ström, and T. Ottosson, "On the optimality of the binary reflected gray code," IEEE Transactions on Information Theory, vol. 50, no. 12, pp. 3170-3182, 2004.

[10] — " "Gray coding for multilevel constellations in gaussian noise," IEEE Transactions on Information Theory, vol. 53, no. 1, pp. 224-235, 2007.

[11] "Digital video broadcasting; second generation framing structure, channel coding and modulation systems for broadcasting, interactive services, news gathering and other broadband satellite applications (DVB-S2)," Tech. Rep., 2009.

[12] "Newtec EL470 IP satellite modem," Tech. Rep., 2009.

[13] G. Karam and H. Sari, "A data predistortion technique with memory for QAM radio systems," IEEE Transactions on Communications, vol. 39, no. 2, pp. 336-344, 1991.

[14] E. Casini, R. De Gaudenzi, and A. Ginesi, "DVB-S2 modem algorithms design and performance over typical satellite channels," Intern. J. of Satellite Communications and Networking, vol. 22, no. 3, pp. 281-318, May/June 2004.

[15] H. A. Aksu and M. Salehi, "Joint optimization of TCQ-TCM systems," IEEE Transactions on Communications, , vol. 44, no. 5, pp. 529-533, May 1996.

[16] G. Foschini, R. Gitlin, and S. Weinstein, "Optimization of twodimensional signal constellations in the presence of gaussian noise," IEEE Transactions on Communications, , vol. 22, no. 1, pp. 28-38, Jan 1974. 